Asia Pac. J. Math. 2022 9:5

ASIA PACIFIC ACADEMIC

\title{
A MATHEMATICAL MODEL OF THE SPREAD AND CONTROL OF NOVEL CORONAVIRUS DISEASE WITH POST-RECOVERY SYMPTOMS
}

\author{
PHILIP N.A. AKUKA ${ }^{1, *}$, BABA SEIDU² ${ }^{2}$ C. S. BORNAA ${ }^{3}$ \\ ${ }^{1}$ Department of Mathematics, Bongo Senior High School, Bongo UE/R, Ghana \\ ${ }^{2}$ Department of Mathematics, School of Mathematical Sciences, \\ C. K. Tedam University of Technology and Applied Sciences, Navrongo UE/R, Ghana \\ ${ }^{3}$ Department of Mathematics and ICT Education, School of Science, Mathematical and Technology Education, \\ C. K. Tedam University of Technology and Applied Sciences, Navrongo UE/R, Ghana \\ *Corresponding author: emmyphil05@gmail.com \\ Received Nov. 22, 2021

\begin{abstract}
Авstract. A Susceptible, Exposed, Infected and Recovered (SEIR) compartment model is proposed to explain the COVID-19 outbreak with postinfection symptoms. Data from Ghana Health Service and sources from published literature are used to estimate the parameters and to perform numerical simulation. It is shown that the model has asymptotically stable disease-free equilibrium and unstable endemic equilibrium points whenever $\mathcal{R}_{0} \leq 1$. The basic reproduction number $\left(\mathcal{R}_{0}\right)$ was found to be 3.27322. Numerical simulation of the model is also done to illustrate the analytical results established. Numerical simulations reveal that an effective strategy to drastically reduce the spread of COVID-19 in Ghana is by mandatory quarantine. 2010 Mathematics Subject Classification. 92D30, 37N25, 34D20, 92B05, 92D25.
\end{abstract}

Key words and phrases. epidemiology; stability; sensitivity; quarantine; post-Covid symptoms.

\section{InTRODUCTION}

Diseases have been part and parcel of the human environment. Research has revealed the etiology of some human diseases but until now, there are myriads of diseases associated with man with unidentified etiology. A viral origin has been suggested for many of these diseases, emphasizing the importance of a continuous search for new viruses [1]. According to [2] "Coronaviruses, a genus of the Coronaviridae family, are enveloped viruses with a large

DOI: $10.28924 / \mathrm{APJM} / 9-5$

(2022 Asia Pacific Journal of Mathematics 
plus-strand RNA genome. The genomic RNA is 27-32 kb in size, capped and polyadenylated". Coronaviruses have been identified in "mice, rats, chicken, turkeys, swine, dogs, cats, rabbits, horses, cattle, and humans, and can cause a variety of severe diseases including gastroenteritis and respiratory tract diseases" [3]. In December 2019, a new coronavirus (COVID-19) was diagnosed in three patients with pneumonia connected to a cluster of acute respiratory illness cases from Wuhan, China. By the end of February 2020, many other countries were experiencing local transmission as a result of importation of the virus. According to [4] "recent comparisons of the genetic sequences of this virus and bat coronaviruses show a $96 \%$ similarity. This is the third zoonotic human coronavirus emerging in the current century, after the severe acute respiratory syndrome coronavirus (SARS-CoV-2) in 2002 that spread to 37 countries and the Middle East respiratory syndrome coronavirus (MERS-CoV) in 2012 that spread to 27 countries". The virus is spread through human-to-human (that is, close contact of host-tosusceptible through coughing, hand shake, and sneezing) and environment-to-human (that is, infected surfaces such as door holding, curtains, cutlery among others). Infected people develop symptoms such as dry cough, fever, fatigue, breathing difficulty, and bilateral lung infiltration in severe cases, similar to those caused by SARS-CoV and MERS-CoV infections [5]. People may develop some post-covid symptoms such as erectile dysfunction, dyspnea , chest pain, and cognitive disturbances, among others. On 11th February, 2020, WHO announced a name for the new coronavirus: COVID-19. On March 11, 2020, the World Health Organization (WHO) formally declared the global outbreak as a pandemic as a result of alarming levels of spread and severity, pointing to over 118,000 cases of coronavirus illness in over 110 countries and territories around the world and the sustained risk of further global spread [6]. Since then, the world has recorded 198 million cases, 179 million recoveries, and 4.2 million deaths. Ghana has not been left out of the virus and is one of the countries with the most infected cases in Africa. Ghana's Ministry of Health confirmed the first two cases of the coronavirus (COVID-19) on Thursday, March 13, 2020. According to authorities, the individuals had returned to Ghana from Norway and Turkey. Ghana has since recorded 103,000 cases, 97,213 recoveries, and 823 deaths. Thus, the management of it since the beginning of the pandemic has been very speculative and most of the treatment options proposed were empirical as no data existed for this rare form of coronavirus. Currently in Ghana, the combination of azithromycin, vitamin $\mathrm{C}$ and zinc among other fever and pain relieve medications seems to be working. 
Several measures had already been instituted to contain the pandemic within more epidemic and endemic areas. Among cross-border measures were local, regional and later international travel bans. Individual and collective preventive measures include social distancing, use of the facemask and personal hygiene through frequent hand washing and use of appropriate sanitizers. [5] findings showed that the virus may be spread via the environment. Anytime those persons infected sneeze or cough without covering their nose or mouth, they are more likely to transmit the virus to the environment via the respiratory droplets that will come out, which can lead to infecting other persons coming into contact with that vicinity. This kind of transmission would occur in the initial days of the virus breakout when the populace would not be aware of the infection threat. Moreover, persons infected certainly would not be isolated, and the majority of the persons would not wear face or nose masks.

[7], came out with SEIRV mathematical model for COVID-19 in Wuhan taking into account environmental factors. The study focused on the "possibility that the virus may survive in the environment for several days, increasing the risk of contamination via surfaces and fomites". Such environmental survival was also confirmed for SARS-CoV [8]. The current study, focused on the assessment of 22 kinds of coronaviruses [9] revealed "that coronaviruses such as SARS$\mathrm{CoV}, \mathrm{MERS}-\mathrm{CoV}$, and endemic human coronaviruses can persist on inanimate surfaces like metal, glass or plastic for up to 9 days, showing strong evidences for the virus' environmental survival". This SEIRV model did not consider vaccination and isolation factors as parameters in the model. Therefore, [10] "research build, analyze using next generation matrix method and simulate the SEIR model through isolation and vaccination on the transmission of coronavirus (COVID-19)". Despite efforts being made to curtail the spread of the COVID-19 disease, the infectious disease is still endemic in many parts of the world including Ghana. The disease has continued causing both health and economic problems nationwide. Researchers have sought to develop strategies to curb the spread and minimize the impact of Covid-19. While these models are numerous and are insightful, the consideration of quarantine in models which consider postinfection symptoms appears unattended to. As a result, this research aims to formulate a mathematical model for the spread and control of COVID-19 with quarantine and post-covid symptoms in Ghana.

\section{Model Formulation}

The deterministic model approach is used to develop the mathematical model for COVID19 transmission dynamics. The total human population is divided into six classes, namely, 
Susceptible S(t), Exposed (infected but not infectious) E(t), Quarantined $Q(t)$, Infected I(t), Recovered $\mathrm{R}(\mathrm{t})$ and individuals with Post-covid symptoms $P_{c}(\mathrm{t})$. The total human population is thus given by $N(t)=S(t)+E(t)+Q)(t)+I(t)+P_{c}(t)+R(t)$.

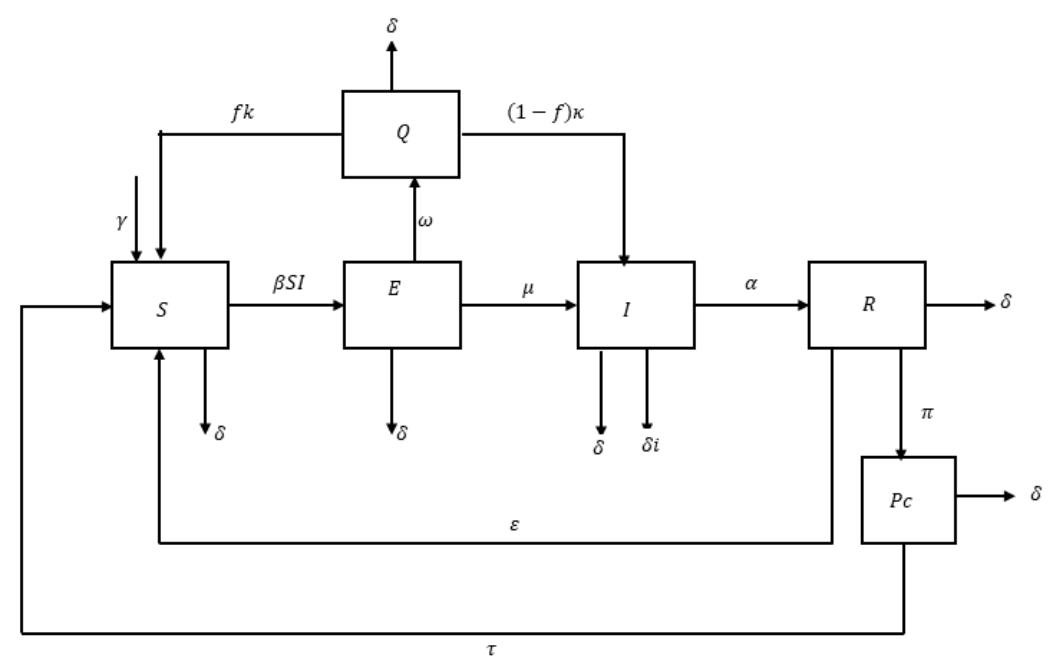

FIGURE 1. Transport diagram for SEIR Model with Quarantined and Post-Covid Symptoms

People enter the susceptible class through recruitment ( by birth or immigration) at a rate $\gamma$. Through mass action, susceptibles come into contact with the infected and are exposed at $\beta S I$. Natural death rate is taken to be $\delta$, while the COVID-19 induced death rate is $\delta_{i}$. People who are exposed are quarantined at a rate $\omega$, and we assume that the proportion $f$ of the quarantined remain susceptible and join the susceptible class at rate $f \kappa$ while the remainder join the infected class at rate $(1-f) \kappa$. People who are exposed become infectious at rate $\mu E$ and those who recover from the disease lose their immunity and move back to the susceptible class at rate $\varepsilon R$. Figure 1 represents the flow diagram of the compartmental model under study.

The model under study is thus given by the following set of ODEs:

$$
\begin{aligned}
& \frac{\mathrm{d} S}{\mathrm{~d} t}=\gamma+\varepsilon R+\tau P_{c}+f \kappa Q-(\delta+\beta I) S \\
& \frac{\mathrm{d} E}{\mathrm{~d} t}=\beta I S-(\omega+\delta+\mu) E \\
& \frac{\mathrm{d}}{\mathrm{d} t}=\mu E+\kappa(1-f) Q-\left(\alpha+\delta+\delta_{i}\right) I \\
& \frac{\mathrm{d} Q}{\mathrm{~d} t}=\omega E-(\delta+\kappa) Q \\
& \frac{\mathrm{d} P_{c}}{\mathrm{~d} t}=\pi R-(\tau+\delta) P_{c} \\
& \frac{\mathrm{d} R}{\mathrm{~d} t}=\alpha I-(\delta+\varepsilon+\pi) R
\end{aligned}
$$


Which gives

$\mathrm{S}(\mathrm{t})+\mathrm{E}(\mathrm{t})+\mathrm{Q}(\mathrm{t})+\mathrm{I}(\mathrm{t})+\mathrm{R}(\mathrm{t})+P_{c}(\mathrm{t})=\mathrm{N}(\mathrm{t})$

With initial conditions,

$\left(\mathrm{S}(0), \mathrm{E}(0), \mathrm{I}(0), \mathrm{Q}(0), \mathrm{R}(0), P_{c}(0)\right) \in \mathbb{R}_{+}^{6}$.

The parameters in the model are further described in Table 1.

\begin{tabular}{|cl|}
\hline PARAMETER & DESCRIPTION \\
\hline$\gamma$ & Recruitment rate of new susceptibles \\
$\delta$ & Rate at which susceptible individuals become exposed by infected individuals and enter exposed class \\
$\delta_{i}$ & Covid-induced death rate of infected individuals \\
$\mu$ & Rate at which individuals leaves the exposed class by becoming infectious \\
$\omega$ & Rate at which exposed individuals are quarantined \\
$\kappa$ & Rate at which exposed individuals who are quarantined revert to susceptible class or join the infected class \\
$f$ & Proportion of the quarantined persons who join the susceptibles \\
$\alpha$ & Rate at which infected individuals leave infected class and enter recovered class. \\
$\varepsilon$ & Rate at which recovered individuals lose temporary immunity and enter susceptible class \\
$\pi$ & Rate at which recovered individuals develop post covid symptoms \\
$\tau$ & Rate at which individuals leaves the post covid symptoms to susceptible class \\
\hline
\end{tabular}

TAble 1. Description of Model Parameters

\section{Qualitative Properties}

3.1. Positivity and Boundedness of Model Solution. The following result relates to the positivity and boundedness of solutions of model (1).

\subsubsection{Boundedness of solution.}

Lemma 3.1. The solution of system (1) is feasible for all $t>0$ if they enter the bounded region $\Omega$

Proof. Let $\Omega=\left(\mathrm{S}, \mathrm{E}, \mathrm{I}, \mathrm{Q}, \mathrm{R}, P_{c}\right) \in \mathbb{R}_{+}^{6}$ be any solution of the system (1) with nonnegative initial conditions.

Moreover, let $\mathrm{N}=\mathrm{S}+\mathrm{E}+\mathrm{I}+\mathrm{Q}+\mathrm{R}+P_{c}$, be the total human population.

Then it implies that

$$
\frac{d N}{d t}=\gamma-\delta N-\delta_{i} I
$$

Using the initial conditions at $\mathrm{t}=0, \mathrm{~N}(0)=N_{0}$;

$$
N_{0} \leq \frac{\gamma}{\delta}+\mathcal{C} \Rightarrow N_{0}-\frac{\gamma}{\delta} \leq \mathcal{C}
$$




$$
\begin{gathered}
N(t) \leq \frac{\gamma}{\delta}+\left(N_{0}-\frac{\gamma}{\delta}\right) e^{-\delta t} \\
\lim _{t \rightarrow \infty} N(t) \leq \frac{\gamma}{\delta}
\end{gathered}
$$

Hence

$$
\Omega=\left\{\left(S, E, I, Q, P_{c}, R\right) \in \mathbb{R}_{+}^{6} \mid 0 \leq N(t) \leq \frac{\gamma}{\delta}\right\}
$$

Therefore, the region $\Omega$ is bounded (i.e., the solutions remain positive for all times, $t$ ) and the model is biologically meaningful and mathematically well-posed in the domain $\Omega$.

\subsubsection{Positivity of solution.}

Lemma 3.2. Let the initial data be $\left\{S(0)>0, E(0), I(0), Q(0), R(0), P_{c}(0) \geq 0\right\} \in \Omega$. Then the solution set $\left\{S, E, I, Q, P_{c}, R\right\}(t)$ of the system is positive for all $t>0$.

Proof. It is easy to show that the following inequalities hold

$$
\begin{aligned}
& S(t) \geq S(0) e^{-(\delta+\beta I) t} \geq 0 \\
& E(t) \geq E(0) e^{-(\omega+\delta+\mu) t} \geq 0 \\
& I(t) \geq I(0) e^{-\left(\alpha+\delta+\delta_{i}\right) t} \geq 0 \\
& Q(t) \geq Q(0) e^{-(\delta+\kappa) t} \geq 0 \\
& R(t) \geq R(0) e^{-(\delta+\varepsilon) t} \geq 0 \\
& P_{c}(t) \geq P_{c}(0) e^{-(\tau+\delta) t} \geq 0
\end{aligned}
$$

Hence, the equations of the model have positive solutions, completing the proof.

3.2. Equilibrium points of the model. Under this, two equilibrium states will be considered, the disease-free equilibrium (where $I=0$ ) and the endemic equilibrium (where $I \neq 0$ ). To determine the equilibrium points, we set the right hand side of the model equations to zero and find the state variables. That is,

$$
\begin{aligned}
& \gamma+\varepsilon R+\tau P_{c}+f \kappa Q-(\delta+\beta I) S=0 \\
& \beta I S-(\omega+\delta+\mu) E=0 \\
& \mu E+\kappa(1-f) Q-\left(\alpha+\delta+\delta_{i}\right) I=0 \\
& \omega E-(\delta+\kappa) Q=0 \\
& \pi R-(\tau+\delta) P_{c}=0 \\
& \alpha I-(\delta+\varepsilon+\pi) R=0
\end{aligned}
$$


3.2.1. Disease free equilibrium point. At disease-free equilibrium, there is no disease in the system, therefore substituting $\mathrm{E}=\mathrm{I}=0$ into the above equations will yield

$$
\begin{gathered}
\gamma-\delta S=0 \\
\Rightarrow S=\frac{\gamma}{\delta}
\end{gathered}
$$

The disease-free equilibrium is then given by $\mathcal{E}_{0}=\left(\frac{\gamma}{\delta}, 0,0,0,0,0\right)$

3.2.2. Endemic equilibrium point. Solution of the system of Equation (1) gives the endemic equilibrium point as $\mathcal{E}^{*}=\left(S^{*}, E^{*}, I^{*}, Q^{*}, P_{c}^{*}, R^{*}\right)$, where

$$
\left.\begin{array}{l}
S^{*}=\frac{1}{(\delta+\beta I)}\left\{\gamma+\left[\left(\frac{\alpha \varepsilon}{(\delta+\varepsilon)\left(\alpha+\delta+\delta_{i}\right)}\right)\left(\mu+\frac{\omega \kappa(1-f)}{\delta+\kappa}\right)+\left(\frac{f \kappa \omega}{\delta+\kappa}\right)+\frac{1}{(\tau+\delta)}\left(\frac{\alpha \pi}{(\delta+\varepsilon+\pi)\left(\alpha+\delta+\delta_{i}\right)}\right)\left(\mu+\frac{\omega \kappa(1-f)}{\delta+\kappa}\right)\right] E^{*}\right\} \\
E^{*}=\frac{\gamma \zeta_{1}-\delta \zeta_{3}}{\zeta_{3} \zeta_{4}-\zeta_{1} \zeta_{2}} \\
I^{*}=\frac{1}{\left(\alpha+\delta+\delta_{i}\right)}\left(\mu+\frac{\omega \kappa(1-f)}{\delta+\kappa}\right) E^{*} \\
Q^{*}=\left(\frac{\omega}{\delta+\kappa}\right) E^{*} \\
P_{c}^{*}=\frac{1}{(\tau+\delta)}\left(\frac{\alpha \pi}{(\delta+\varepsilon+\pi)\left(\alpha+\delta+\delta_{i}\right)}\right)\left(\mu+\frac{\omega \kappa(1-f)}{\delta+\kappa}\right) E^{*} \\
R^{*}=\left(\frac{\alpha \kappa(1-f)}{\delta+\varepsilon)\left(\alpha+\delta+\delta_{i}\right)}\right)\left(\mu+\frac{\omega+\kappa}{\delta+\kappa} E^{*}\right.
\end{array}\right\}
$$

where

$$
\begin{aligned}
& \zeta_{1}=\beta[\mu(\delta+\kappa)+\omega \kappa(1-f)] \\
& \zeta_{2}=\left[\left(\frac{\alpha \varepsilon}{(\delta+\varepsilon)\left(\alpha+\delta+\delta_{i}\right)}\right)\left(\mu+\frac{\omega \kappa(1-f)}{\delta+\kappa}\right)+\frac{f \kappa \omega}{\delta+\kappa}+\frac{1}{(\tau+\delta)}\left(\frac{\alpha \pi}{(\delta+\varepsilon+\pi)\left(\alpha+\delta+\delta_{i}\right)}\right)\left(\mu+\frac{\omega \kappa(1-f)}{\delta+\kappa}\right)\right] \\
& \zeta_{3}=(\omega+\delta+\mu)\left(\alpha+\delta+\delta_{i}\right)(\delta+\kappa) \\
& \zeta_{4}=\frac{\beta}{\left(\alpha+\delta+\delta_{i}\right)}\left(\mu+\frac{\omega \kappa(1-f)}{\delta+\kappa}\right)
\end{aligned}
$$

3.3. Basic Reproduction Number. The basic reproduction number $\left(\mathcal{R}_{0}\right)$ is determined using the Next Generation Matrix (NGM) method. Mathematically, the basic reproduction number, $\mathcal{R}_{0}$ is the dominant eigenvalue of the next generation matrix $[11,12]$. The compartments which are directly involved in the transmission of the disease are only E, Q, I. Therefore, from system 1 we have

$$
\begin{aligned}
& \frac{\mathrm{d} E}{\mathrm{~d} t}=\beta I S-(\omega+\delta+\mu) E \\
& \frac{\mathrm{d} I}{\mathrm{~d} t}=\mu E+\kappa(1-f) Q-\left(\alpha+\delta+\delta_{i}\right) I \\
& \frac{\mathrm{d} Q}{\mathrm{~d} t}=\omega E-(\delta+\kappa) Q
\end{aligned}
$$


The above system is written as $\frac{\mathrm{d} y}{\mathrm{~d} t}=\Theta(y)-\Upsilon(y)$, where $y=\left(\begin{array}{c}E \\ Q \\ I\end{array}\right), \Theta(y)=\left(\begin{array}{l}\beta S I \\ 0 \\ 0\end{array}\right)$,

$\Upsilon(y)=\left(\begin{array}{l}(\omega+\delta+\mu) E \\ (\delta+\kappa) Q-\omega E \\ \left(\alpha+\delta+\delta_{i}\right) I-\mu E-\kappa(1-f) Q\end{array}\right)$

It is clear from system $(1)$ that $\mathcal{E}_{0}=\left(\frac{\gamma}{\delta}, 0,0,0,0\right)$ is a disease free equilibrium. Now the Jacobian matrix of $\Theta$ and $\Upsilon$ at the disease-free equilibrium are, respectively, given by

$$
\mathcal{F}=J\left(\Theta \mid \mathcal{E}_{0}\right)=\left(\begin{array}{ccc}
0 & 0 & \beta S \\
0 & 0 & 0 \\
0 & 0 & 0
\end{array}\right)
$$

and

$$
\mathcal{V}=J\left(\Upsilon \mid \mathcal{E}_{0}\right)=\left(\begin{array}{ccc}
(\omega+\delta+\mu) & 0 & 0 \\
-\omega & (\delta+\kappa) & 0 \\
-\mu & -\kappa(1-f) & \left(\alpha+\delta+\delta_{i}\right)
\end{array}\right)
$$

$\mathcal{R}_{0}$ is the spectral radius of $\mathcal{F} \mathcal{V}^{-1}$ and is given by

$$
\mathcal{R}_{0}=\frac{\gamma \beta}{\delta\left(\alpha+\delta+\delta_{i}\right)}
$$

3.4. Local stability of equilibria. In this section, we investigate the local asymptotic stability of the equilibria.

Theorem 3.1. The disease-free equilibrium $\mathcal{E}_{0}$ of the model is asymptotically stable whenever $\mathcal{R}_{0} \leq 1$ and unstable otherwise.

Proof. The Jacobian matrix at $\mathcal{E}_{0}$ of system (1) is given by

$$
J_{\mathcal{E}_{0}}=\left[\begin{array}{cccccc}
-\delta & 0 & -\beta S & f \kappa & \tau & \varepsilon \\
0 & -(\omega+\mu+\delta) & \beta S & 0 & 0 & 0 \\
0 & \mu & -\left(\alpha+\delta+\delta_{i}\right) & \kappa(1-f) & 0 & 0 \\
0 & \omega & 0 & -(\delta+\kappa) & 0 & 0 \\
0 & 0 & 0 & 0 & -(\delta+\tau) & \pi \\
0 & 0 & \alpha & 0 & 0 & -(\delta+\varepsilon+\pi)
\end{array}\right]
$$

Three eigenvalues are clearly $\lambda_{1}=-\delta, \lambda_{2}=-(\delta+\tau)$ and $\lambda_{3}=-(\delta+\varepsilon+\pi)$, which are negative and the rest are found by solving equation (2), which is the characteristic of the Jacobian 
matrix $J_{\mathcal{E}_{0}}$.

The characteristic equation of system (1) is given by

$$
\lambda^{3}+a_{1} \lambda^{2}+a_{2} \lambda+a_{3}=0
$$

where

$$
\begin{aligned}
& a_{1}=\left(3 \delta+\kappa+\delta_{i}+\alpha+\omega+\mu\right), \\
& a_{2}=\mu\left(1-\mathcal{R}_{0}\right)\left(\alpha+\delta+\delta_{i}\right)+2 \alpha \delta+\alpha \kappa+\alpha \omega+3 \delta^{2}+2 \delta \kappa+\delta \mu+2 \delta \omega \\
& +2 \delta \delta_{i}+\kappa \mu+\kappa \omega+\kappa \delta_{i}+\delta_{i} \omega \\
& a_{3}=\omega \beta S \kappa f+\left(1-\mathcal{R}_{0}\right)\left(\alpha+\delta+\delta_{i}\right)(\delta \mu+\kappa \mu+\kappa \omega)+\delta^{2}\left(\alpha+\delta+\kappa+\omega+\delta_{i}\right) \\
& +\delta\left(\alpha+\delta_{i}\right)(\kappa+\omega)
\end{aligned}
$$

Thus, all coefficients of equation (2) are positive whenever $\mathcal{R}_{0} \leq 1$. Applying Descartes Sign rule, therefore all roots of equation (2) are negative. Therefore, the DFE of model 1 is asymptotically stable whenever $\mathcal{R}_{0} \leq 1$ otherwise it is unstable, concluding the proof.

Theorem 3.2. The Endemic equilibrium $\mathcal{E}^{*}$ is locally asymptotically stable for $\mathcal{R}_{0}>1$

Proof. The Jacobian matrix for the system (1) is given by

$$
J_{\mathcal{E}^{*}}=\left[\begin{array}{cccccc}
-\left(\delta+\beta I^{*}\right) & 0 & -\beta S^{*} & f \kappa & \tau & \varepsilon \\
\beta I^{*} & -(\omega+\mu+\delta) & \beta S^{*} & 0 & 0 & 0 \\
0 & \mu & -\left(\alpha+\delta+\delta_{i}\right) & \kappa(1-f) Q & 0 & 0 \\
0 & \omega & 0 & -(\delta+\kappa) & 0 & 0 \\
0 & 0 & 0 & 0 & -(\delta+\tau) & \pi \\
0 & 0 & \alpha & 0 & 0 & -(\delta+\varepsilon+\pi)
\end{array}\right]
$$

With $\mathrm{A}=\left(\delta+\beta I^{*}\right) ; \mathrm{B}=\beta I^{*} ; \mathrm{C}=(\omega+\mu+\delta) ; \mathrm{D}=\mu ; \mathrm{Z}=\omega ; \mathrm{F}=\beta S^{*} ; \mathrm{G}=\left(\alpha+\delta+\delta_{i}\right) ; \mathrm{Y}=\alpha ; \mathrm{J}=f \kappa$; $\mathrm{K}=\kappa(1-f) Q ; \mathrm{L}=(\delta+\kappa) ; \mathrm{M}=(\delta+\tau) ; \mathrm{N}=\pi ; \mathrm{R}=\tau ; \mathrm{T}=\varepsilon$ and $\mathrm{P}=(\delta+\varepsilon+\pi)$

The characteristic equation of the jacobian matrix $J_{\mathcal{E}^{*}}$ is

$$
\lambda^{6}+a_{1} \lambda^{5}+a_{2} \lambda^{4}+a_{3} \lambda^{4}+a_{5} \lambda^{2}+a_{4} \lambda+a_{6}=0
$$

where

$$
\begin{gathered}
a_{1}=(A+C+G+L+M+P) \\
a_{2}=(M P+(A+C+G+L)(M+P)+A C+G L+(A+C)(G+L)-F D) \\
a_{3}=\begin{array}{l}
(A+C+G+L) M P+(A C+G L+(A+C)(G+L))(M+P)+G L(A+C)+A C(G+L)) \\
-((A F D+F(K Z+D(L+M+P))))+(D F+J Z)
\end{array}
\end{gathered}
$$




$$
\begin{gathered}
(A C G L+(A C+G L+(A+C)(G+L)) M P+(G L(A+C)+A C(G+L)(M+P))) \\
a_{4}=-(A F(K Z+D(L+M+P))+F(K Z(M P+D)(M P+L(M+P)))) \\
+(M+P+L+T Y) D Z+(F K+(G+M+P) J Z) \\
a_{5}=-(A C G L(M+P)+(G L(A+C)+A C(G+L)) M P \\
((M P K Z+D L M P)+A F(K Z(M+P)+D(M P+L(M+P)))) \\
a_{6}=(A C G M P L-A F(M P K Z+D L M P)+D L M P+(M P G J+K Y(R N+T M)) Z)
\end{gathered}
$$

From the Routh-Hurwitz stability criterion if, $a_{i}>0, a_{1} a_{2} a_{3}>a_{3}^{2}+a_{1}^{2} a_{4}$ and $\left(a_{1} a_{4}-\right.$ $\left.a_{5}\right)\left(a_{1} a_{2} a_{3}-a_{3}^{2}-a_{1}^{2} a_{4}\right)>a_{5}\left(a_{1} a_{2}-a_{3}\right)^{2}+a_{1} a_{5}^{2}$, are satisfied, then the system has negative roots and the roots are all stable, otherwise the roots are unstable. Therefore, the endemic equilibrium point is stable when $\mathcal{R}_{0}>1$.

3.5. Sensitivity analysis of $\mathcal{R}_{0}$. Sensitivity analysis plays a vital part in epidemiological models. It enable us to determine which parameters of a model are most responsible for generating the variability in the value of the model's output over time. The impact of each parameter on the basic reproduction number $\left(\mathcal{R}_{0}\right)$ is investigated. This is done using the normalized forward sensitivity index (NFSI).

The normalized forward sensitivity index of $\mathcal{R}_{0}$ with respect to the parameter $\mathrm{x}$ is defined as $[13]$

$$
\Gamma_{x}^{\mathcal{R}_{0}}=\frac{\partial \mathcal{R}_{0}}{\partial x} \times \frac{x}{\mathcal{R}_{0}}
$$

The sensitivity indices are computed and presented in Table 2 using the parameters values in Table 3.

\begin{tabular}{clc}
\hline PARAMETER & DESCRIPTION & SENSITIVITY INDEX \\
\hline$\beta$ & Transmission rate & +1 \\
$\gamma$ & Recruitment rate & +1 \\
$\alpha$ & Rate of recovery & -0.9618 \\
$\delta$ & Natural death rate & -0.3202 \\
$\delta_{i}$ & Covid-19 induced death rate & -0.0139 \\
\hline
\end{tabular}

TABLE 2. Sensitivity indices of $\mathcal{R}_{0}$.

From Table 2, it is seen that increasing (decreasing) $\beta$ or $\gamma$ by say $10 \%$ will lead to an increase ( decrease) in $\mathcal{R}_{0}$. Similarly, when we marginally increase (decrease) $\delta$ or $\alpha$ or $\delta_{i}$ by say $10 \%$, 
$\mathcal{R}_{0}$ will decrease (increase)

Thus, $\beta$ and $\gamma$ are directly related to $\mathcal{R}_{0}$ While $\delta, \alpha$ and $\delta_{i}$ are inversely related to $\mathcal{R}_{0}$.

\section{Numerical Simulation}

4.1. Parameter Estimation. We estimate the parameters of the model based on COVID-19 data from Ghana Health Service and sources from published literature. Since the period generally recommended for quarantine of people suspected of being exposed to COVID-19 is 14 days [14], we set the rate at which quarantine susceptible individuals revert to the non-quarantined susceptible class $(\kappa)$ to be $\kappa=\frac{1}{10}$ per day. Some studies have estimated the incubation period for COVID-19 to range from 2-14 days, with about $97.5 \%$ of infected people developing disease symptoms within 11.5 days of infection [15]. We consider an average incubation period(taken from these ranges) of 5.75 days, so that $\mu=\frac{1}{5.75}$ per day [15]. The transmission rate $(\beta)$ is given as $\beta=\frac{\text { effective contact }}{\text { total contact }}=0.0369$ per day. The natural death rate of Ghana stands at 7.75 deaths per 1000 population hence we have $\delta=0.0078$ per day. The parameter estimates are summarized in Table 3.

\begin{tabular}{clcl}
\hline PARA. & DESCRIPTION & VALUE (per day) & SOURCE \\
\hline$\kappa$ & Rate at which exposed become susceptible or infected & 0.1 & Estimated from [14] \\
$\mu$ & Rate of exposed person to infectious & 0.1739 & Estimated from [15] \\
$\alpha$ & Rate of recovery & 0.310 & Assumed \\
$\beta$ & Transmission rate & 0.0369 & Estimated from GHS \\
$\delta$ & Natural death rate & 0.0078 & Estimated from GSS \\
$\delta_{i}$ & Covid-19 induced death rate & 0.0045 & Estimated from GHS \\
$\varepsilon$ & Immunity loss rate & 0.0167 & Assumed \\
$\omega$ & Rate at which exposed persons are quarantined & 0.173 & Assumed \\
$\pi$ & Rate at which recovered persons develop post-covid symptoms & 0.015 & Assumed \\
$\gamma$ & Recruitment rate & 0.223 & Assumed \\
$f$ & Proportion of quarantined persons to susceptibles & 0.0648 & Assumed \\
$\tau$ & Rate at which post-covid symptoms move to susceptible class & 0.0125 & Assumed \\
\hline
\end{tabular}

TABle 3. Parameters estimates of the model for COVID-19 
4.2. Simulations. To show numerically the impact of the controls $\beta$ and $\omega$ had the model (1) is solved for varying values of each control, keeping all other parameters constant. The results of the simulations are presented in Figures 2- 4.
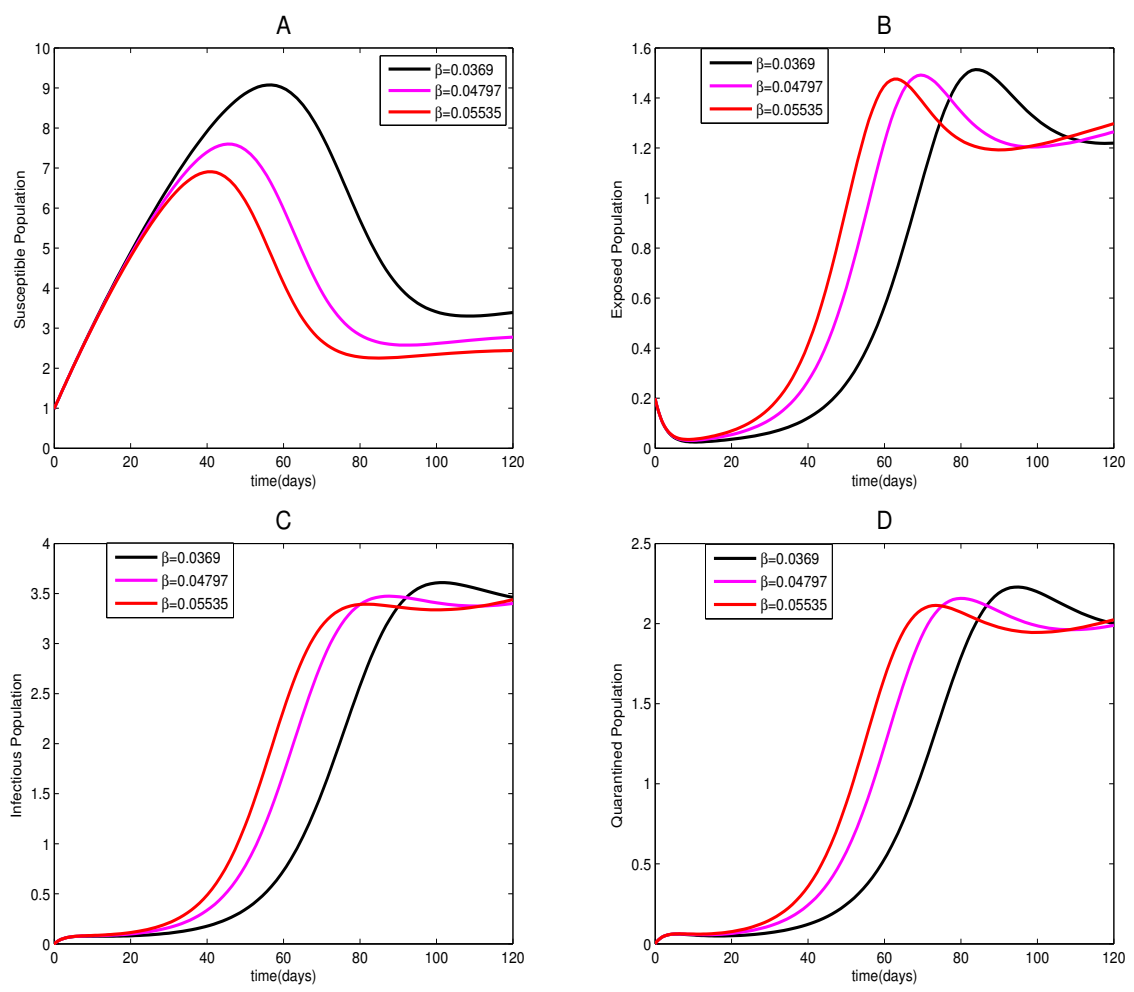

FIGURE 2. Solution curves depicting the impact of $\beta$ on each of the classes

It is observed from Figure 2(B, C and D) that an increase in $\beta$ will lead to more Susceptibles getting exposed, which will lead to increase in infected and quarantined persons and a decrease in the susceptibles in Figure 2A. Therefore, efforts needed to reduce $\beta$ such as mandatory quarantine should be made. 

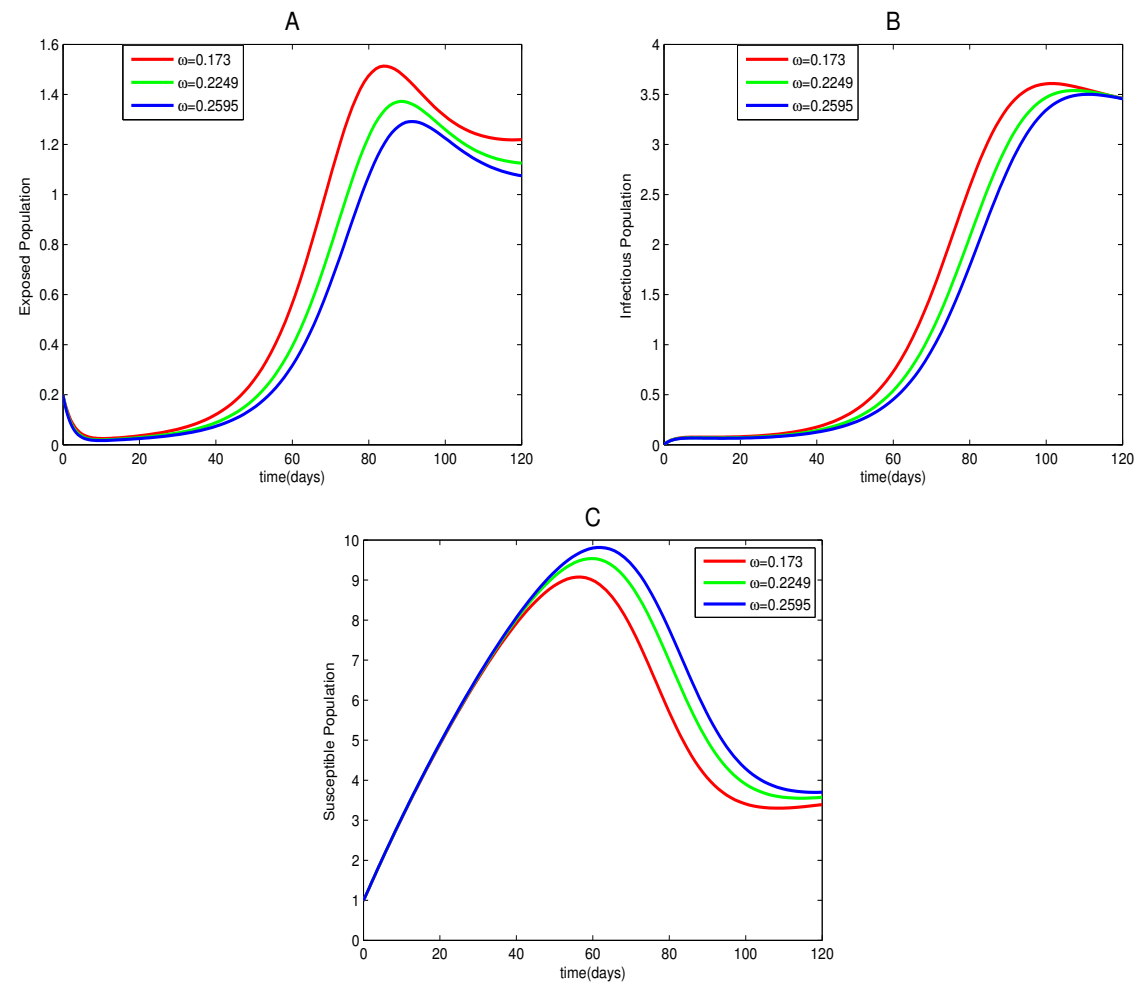

FIGURE 3. Solution curves depicting the impact of $\omega$ on each of the classes

In Figure 3C, it is observed that as the quarantined rate, $\omega$ increased, and then the susceptible population is increased. It leads to a decrease in the exposed and infective population in Figure 3(A and B), respectively. The impact of $\omega$ is opposite to that of $\beta$. Therefore, attempts at increasing $\omega$ should be made. A better approach to reduce the spread of COVID-19 disease should include measures aimed at reducing $\beta$ and increasing $\omega$. 

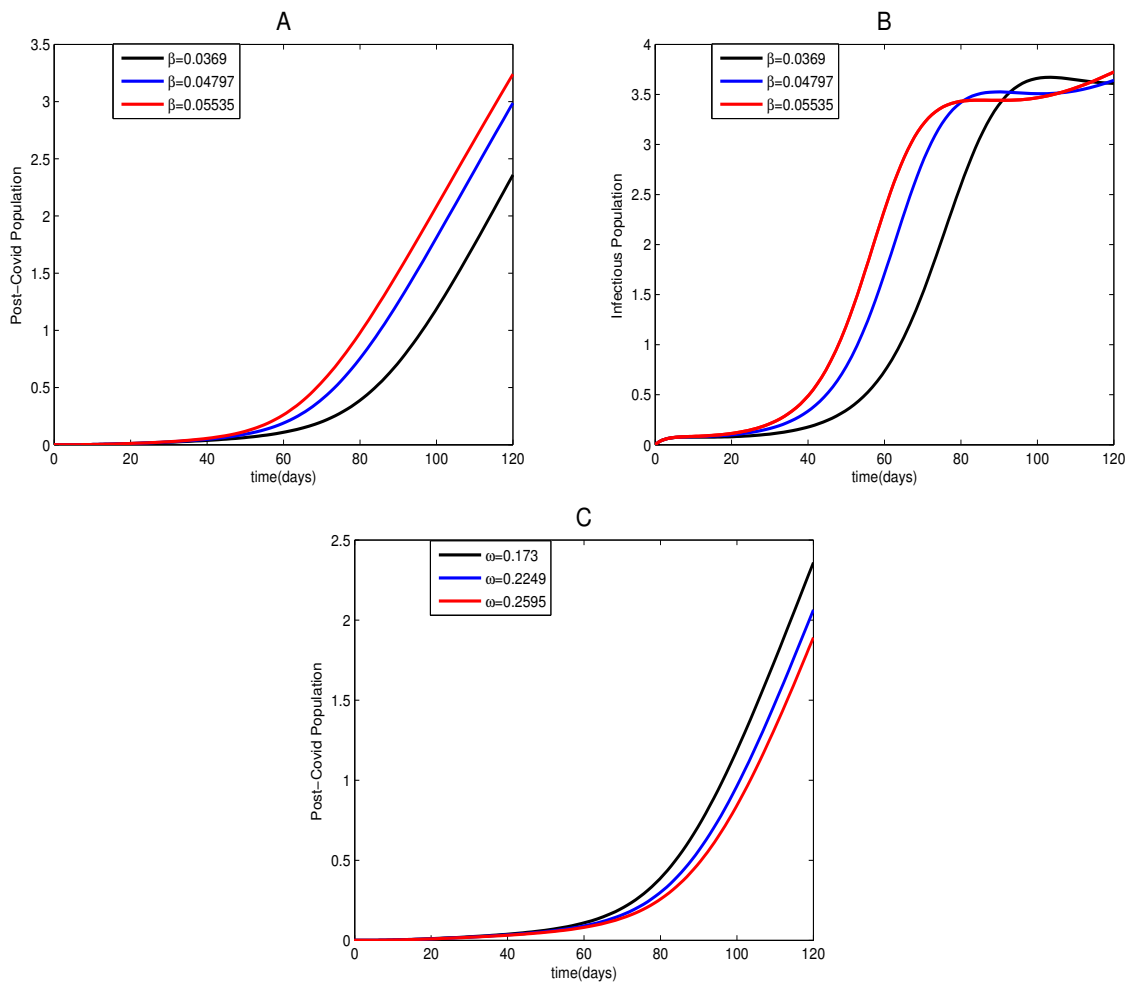

FIGURE 4. Solution curves depicting the impact of $\omega$ and $\beta$ on each of the classes

It is observed from Figure 4(A and B) that an increase in $\beta$ will lead to more susceptibles getting exposed, which will lead to an increase in infected persons and subsequently an increase in postinfection symptoms. In Figure 4C, it is observed that as the quarantined rate $\omega$ increases, then the post-covid population decreases. Therefore, efforts needed to reduce $\beta$ such as mandatory quarantine should be made.

\section{Discussion And Conclusions}

5.1. Discussions. A mathematical model for the spread and control of COVID-19 with PostCovid Symptoms in Ghana is formulated and analyzed. The main objective of this study was to assess the impact of quarantine on the transmission dynamics of the COVID-19. We calculated the basic reproduction number $\mathcal{R}_{0}$ using the next generation matrix for the model. If $\mathcal{R}_{0}<1$, the disease cannot persist in the country and when $\mathcal{R}_{0}>1$, the disease can persist. The equilibrium points were obtained and their stability established. We proceeded to simulate our model for various values of $\beta$ and $\omega$ and observed the changes that occurred in the model results.

It is found in Figure(4) B, C and D that an increase in the transmission $\beta$ will lead to more susceptibles getting exposed, which will lead to an increase in infected and quarantine persons. 
Also, it is found in Figure(3) $\mathrm{C}$ that, an increase in the quarantine rate $\omega$ will increase the susceptible population and will lead to a decrease in the exposed and infected population in Figure(3) A and B. From Figure(4) A and B, it is seen that as the transmission rate increase, more people are infected and subsequently an increase in the number of post-infection symptoms. However, with the implementation of mandatory quarantine, the post-infection symptoms population decrease as seen in Figure(4) C. The following results were obtained from the analysis of the models. Based on the estimated parameter values for our model, the basic reproduction number $\mathcal{R}_{0}=3.27322$, it implies that on average, each infectious individual transmits the virus to 3 people; since $\mathcal{R}_{0}>1$ an outbreak of COVID-19 will result in an epidemic in Ghana. The disease-free equilibrium point is $(28.58974,0,0,0,0)$ and the endemic equilibrium point is $(4.4068,0.8998,2.4767,1.4440,7.2178)$.

Disease management is concerned with lowering the basic reproduction number to a value less than unity. In this case, reduction of the basic reproduction number, $\mathcal{R}_{0}$ to a value less than unity involves reducing the transmission rate $\beta$. The numerical analysis of the models indicated that the most effective way for eliminating COVID-19 is through quarantine of exposed individuals as found in Figure 3. This study corresponds with [16] suggestion that quarantine is an effective measure for controlling infectious disease and [17]'s research which suggest that quarantine and isolation as control strategies for COVID-19 outbreak. It has also been found that, after persons recover from the disease, some of them develop some post-covid symptoms (such as erectile dysfunction, dyspnea, chest pain , cognitive disturbances among others). The sensitivity analysis revealed that an effective strategy to drastically reduce the spread of COVID-19 in Ghana is by mandatory quarantine.

5.2. Conclusion. This model focused on the control and spread of COVID-19 with post-covid symptoms in Ghana through a deterministic approach.

We first prove that there exists a domain, $\Omega=\left\{(S, E, I, Q, R) \in \mathbb{R}_{+}^{6} \mid 0 \leq N(t) \leq \frac{\gamma}{\delta}\right\}$ where the model is epidemiologically meaningful and mathematically well-posed. The basic reproductive number, $\mathcal{R}_{0}$ of our SQEIR model was calculated to be 3.27322, which means that on average, an infective person can transmit the virus to 3 people. The stability analysis of the disease-free equilibrium has been found to be unstable; hence, we conclude that the disease will spread. However, the stability analysis of the endemic equilibrium has been found to be unstable, hence, the disease will not persist. The sensitivity analysis that was carried out on $\mathcal{R}_{0}$ showed that the transmission rate, $\beta$ has 
a great impact on the spread of COVID-19 in Ghana. Changing the value of $\beta$ at the same rate as the other parameter values increases the proportions of both the exposed and infected population. We conclude that COVID-19 transmission is as a result of mass action (that is, the interaction between active COVID-19 persons and the susceptible class). To reduce the transmission rate, we introduced a mandatory quarantined rate, $\omega$ which decreased the exposed and infected class. The numerical analysis of the model suggested that the most effective strategy for controlling COVID-19 in Ghana is the use of mandatory quarantine. Hence, the impact of reducing the transmission rate, $\beta$ using quarantined rate, $\omega$ has a great impact in reducing the transmission of disease and disease related deaths in Ghana.

It was also found out that, as more people recovered from the disease, instead of the susceptible population increasing, it was rather decreasing due to the result that some recovered persons developed post-covid symptoms such as erectile dysfunction, dyspnea, chest pain, and cognitive disturbances, among others. The results obtained can be used as a reference for slowing the spread of COVID-19 in Ghana. The following measures are therefore recommended to reduce the spread of COVID-19 virus disease:

(1) The spread of COVID-19 disease can be eliminated in Ghana by reducing the transmission rate $\beta$ through quarantine $\omega$ as seen in Figure 3. Therefore, immigrants should be made to go through a mandatory quarantine as that is the latency period of the virus.

(2) Quarantine people who are exposed while a clinical test is carried out on them so that when their results are out, there will not be the need to do contact tracing if they are tested positive.

These will help the government and health authorities to mitigate the spread of COVID-19 in Ghana.

\section{DATA AVAILABILITY STATEMENT}

The parameter values (data) used to support the findings of this study have been described in subsection 4.1 .

\section{REFERENCES}

[1] [1]S.A. Stohlman, D.R. Hinton, Viral induced demyelination, Brain Pathol. 11 (2006), 92-106. https: //doi.org/10.1111/j.1750-3639.2001.tb00384.x.

[2] K.V. Holmes, SARS coronavirus: a new challenge for prevention and therapy, J. Clin. Invest. 111 (2003), 1605-1609. https://doi.org/10.1172/JCI18819. 
[3] J.S. Guy, J.J. Breslin, B. Breuhaus, S. Vivrette, L.G. Smith, Characterization of a coronavirus isolated from a diarrheic foal, J. Clin. Microbiol. 38 (2000), 4523-4526. https: //doi .org/10 . 1128/ JCM . 38.12 .4523-4526. 2000.

[4] P. Zhou, X.-L. Yang, X.-G. Wang, et al., Discovery of a novel coronavirus associated with the recent pneumonia outbreak in humans and its potential bat origin, BioRxiv (2020). https://doi.org/10.1101/2020. 01.22 .914952$.

[5] L.E. Gralinski, V.D. Menachery, Return of the Coronavirus: 2019-nCoV, Viruses. 12 (2020), 135. https: //doi.org/10.3390/v12020135.

[6] The WHO Just Declared Coronavirus COVID-19 a Pandemic (2020). https://time.com/5791661/ who-coronavirus-pandemic-declaration/

[7] C. Yang, J. Wang, A mathematical model for the novel coronavirus epidemic in Wuhan, China, Math. Biosci. Eng. 17 (2020), 2708-2724. https://doi.org/10.3934/mbe. 2020148.

[8] C. Geller, M. Varbanov, R. Duval, Human Coronaviruses: insights into environmental resistance and its influence on the development of new antiseptic strategies, Viruses. 4 (2012), 3044-3068. https : //doi .org/ $10.3390 / \mathrm{v} 4113044$.

[9] G. Kampf, D. Todt, S. Pfaender, E. Steinmann, Persistence of coronaviruses on inanimate surfaces and their inactivation with biocidal agents, J. Hosp. Infect. 104 (2020), 246-251. https://doi .org/10.1016/j . jhin. 2020.01 .022$.

[10] S. Annas, Muh. Isbar Pratama, Muh. Rifandi, W. Sanusi, S. Side, Stability analysis and numerical simulation of SEIR model for pandemic COVID-19 spread in Indonesia, Chaos Solitons Fractals. 139 (2020), 110072. https://doi.org/10.1016/j.chaos.2020.110072.

[11] O. Diekmann, J.A.P. Heesterbeek, M.G. Roberts, The construction of next-generation matrices for compartmental epidemic models, J. R. Soc. Interface. 7 (2010), 873-885. https://doi .org/10.1098/rsif . 2009. 0386.

[12] P. van den Driessche, J. Watmough, Reproduction numbers and sub-threshold endemic equilibria for compartmental models of disease transmission, Math. Biosci. 180 (2002), 29-48. https://doi . org/10. 1016/S0025-5564 (02)00108-6.

[13] J.K.K. Asamoah, Z. Jin, G.-Q. Sun, M.Y. Li, A deterministic model for Q fever transmission dynamics within dairy cattle herds: using sensitivity analysis and optimal controls, Comput. Math. Meth. Med. 2020 (2020), 6820608.

[14] J.F.-W. Chan, S. Yuan, K.-H. Kok, et al., A familial cluster of pneumonia associated with the 2019 novel coronavirus indicating person-to-person transmission: a study of a family cluster, The Lancet. 395 (2020), 514-523. https://doi.org/10.1016/S0140-6736(20)30154-9.

[15] S.A. Lauer, K.H. Grantz, Q. Bi, et al., The incubation period of coronavirus disease 2019 (COVID-19) from publicly reported confirmed cases: estimation and application, Ann. Internal Med. 172 (2020), 577-582. https://doi.org/10.7326/M20-0504. 
[16] B. Tang, F. Xia, S. Tang, N.L. Bragazzi, Q. Li, X. Sun, J. Liang, Y. Xiao, J. Wu, The effectiveness of quarantine and isolation determine the trend of the COVID-19 epidemics in the final phase of the current outbreak in China, Int. J. Infect. Dis. 95 (2020), 288-293. https : //doi.org/10.1016/j .i jid. 2020 .03.018.

[17] Z. Memon, S. Qureshi, B.R. Memon, Assessing the role of quarantine and isolation as control strategies for COVID-19 outbreak: A case study, Chaos Solitons Fractals. 144 (2021), 110655. https://doi .org/10. $1016 / j$. chaos . 2021.110655. 